\title{
UHRF genes regulate programmed interdigital tissue regression and chondrogenesis in the embryonic limb
}

\author{
Cristina Sanchez-Fernandez ${ }^{1}$, Carlos I. Lorda-Diez', Juan A. García-Porrero', Juan A. Montero' and Juan M. Hurlé
}

\begin{abstract}
The primordium of the limb contains a number of progenitors far superior to those necessary to form the skeletal components of this appendage. During the course of development, precursors that do not follow the skeletogenic program are removed by cell senescence and apoptosis. The formation of the digits provides the most representative example of embryonic remodeling via cell degeneration. In the hand/foot regions of the embryonic vertebrate limb (autopod), the interdigital tissue and the zones of interphalangeal joint formation undergo massive degeneration that accounts for jointed and free digit morphology. Developmental senescence and caspase-dependent apoptosis are considered responsible for these remodeling processes. Our study uncovers a new upstream level of regulation of remodeling by the epigenetic regulators Uhrf1 and Uhrf2 genes. These genes are spatially and temporally expressed in the pre-apoptotic regions. UHRF1 and UHRF2 showed a nuclear localization associated with foci of methylated cytosine. Interestingly, nuclear labeling increased in cells progressing through the stages of degeneration prior to TUNEL positivity. Functional analysis in cultured limb skeletal progenitors via the overexpression of either UHRF1 or UHRF2 inhibited chondrogenesis and induced cell senescence and apoptosis accompanied with changes in global and regional DNA methylation. Uhrfs modulated canonical cell differentiation factors, such as Sox9 and Scleraxis, promoted apoptosis via up-regulation of Bak1, and induced cell senescence, by arresting progenitors at the $S$ phase and upregulating the expression of p21. Expression of Uhrf genes in vivo was positively modulated by FGF signaling. In the micromass culture assay Uhrf1 was down-regulated as the progenitors lost stemness and differentiated into cartilage. Together, our findings emphasize the importance of tuning the balance between cell differentiation and cell stemness as a central step in the initiation of the so-called "embryonic programmed cell death" and suggest that the structural organization of the chromatin, via epigenetic modifications, may be a precocious and critical factor in these regulatory events.
\end{abstract}

\section{Introduction}

The autopod is a paddle shaped structure consisting of a core of skeletal progenitors covered by the ectoderm. During the course of development, the progenitors aggregate to form radial digit condensations separated by

\footnotetext{
Correspondence: Juan M. Hurlé (hurlej@unican.es)

'Departamento de Anatomía y Biología Celular and IDIVAL, Universidad de Cantabria, 39011 Santander, Spain

These authors contributed equally: Cristina Sanchez-Fernandez, Carlos I. LordaDiez

Edited by J. Chipuk
}

interdigital regions. Cells in the interdigital regions are initially undifferentiated but soon follow massive degeneration that sculpts the digit contours. In chick and mouse embryos the elimination of the interdigital tissue lasts more than $30 \mathrm{~h}$. During this period the interdigital cells retain stemness to respond to differentiation signals to form ectopic digits ${ }^{1}$.

The control of interdigit remodeling has been the subject of intense research. Morphological and molecular analyses revealed the redundant involvement of several degenerative pathways including canonical apoptosis, cell death

\section{(c) The Author(s) 2019}

(c) (i) Open Access This article is licensed under a Creative Commons Attribution 4.0 International License, which permits use, sharing, adaptation, distribution and reproduction c. in any medium or format, as long as you give appropriate credit to the original author(s) and the source, provide a link to the Creative Commons license, and indicate if changes were made. The images or other third party material in this article are included in the article's Creative Commons license, unless indicated otherwise in a credit line to the material. If material is not included in the article's Creative Commons license and your intended use is not permitted by statutory regulation or exceeds the permitted use, you will need to obtain permission directly from the copyright holder. To view a copy of this license, visit http://creativecommons.org/licenses/by/4.0/. 
associated with lysosomal activation ${ }^{2,3}$, and cell senescence ${ }^{4}$. All these degenerative routes are preceded by intense DNA damage $^{5}$, which is suggestive of common upstream regulation. BMPs have been identified as active factors responsible for interdigit regression. However, while the interdigital application of exogenous BMPs in the embryonic limb induces cell death and DNA damage, the same BMPs stimulate cell proliferation and differentiation in the tip of the fingers, that are also constituted by the same skeletal progenitors ${ }^{6}$. This finding suggests that complementary signals cooperate with BMPs to trigger the degenerative events. The hypothesis of the present study is that the embryonic degenerative processes may share regulatory factors occurring in tumor tissues. DNA damage, cell senescence, and apoptosis are associated with epigenetic and chromatin architecture alterations of cancerous cells $^{7}$ that may determine tumorigenesis and sensitivity to chemotherapy and irradiation ${ }^{8,9}$.

In this study, we analyzed whether ubiquitin-like containing plant homeodomain and RING finger domain (UHRF) epigenetic modulators 1 and 2 participate in the degenerative processes associated with digit development. This gene family is functionally associated with DNA damage and participates in the regulation of proliferation and cell survival of numerous malignancies ${ }^{10}$. UHRF proteins are complex factors containing four functional domains that modulate transcriptional regulation via chromatin modifications. Uhrf genes are upregulated in many cancer cells and may behave as either oncogenes or tumor suppressors ${ }^{10}$. Depletion of UHRF1 increases the chemosensitivity of cancer cells to hydroxyurea resistance $^{11}$ and increases their sensitivity to gammairradiation ${ }^{12}$. UHRF2, in turn, has been characterized as a component of the ubiquitin proteasome degradation machinery ${ }^{13}$ with pro-apoptotic functions in oncogenestressed cells ${ }^{14}$. The significance of Uhrf genes in developmental systems has received less attention. Mice and zebrafish deficient in UHRF1 die during the course of development ${ }^{15,16}$, and embryonic stem cells null for UHRF1 are hypersensitive to DNA-damaging agents ${ }^{15}$. Furthermore, Uhrf1 knockout directed to limb mesoderm implicates this protein in appendicular development ${ }^{17}$, as these mice show shortened long bones and dysregulated chondrocyte maturation and proliferation via alterations of the growth plate. Uhrf2 knockout mice are viable and lack morphological defects ${ }^{18}$, but there is evidence of its implication in the pathogenesis of neurodegenerative diseases ${ }^{19}$. Here, we show that Uhrf1 and Uhrf2 genes are expressed in the interdigital mesoderm and interphalangeal joints where undifferentiated cells undergo senescence and apoptosis. At protein level UHRFs associated with zones of DNA methylation. Functional analysis via the overexpression of either UHRF1 or UHRF2 inhibited chondrogenesis and induced cell senescence and apoptosis of cultured limb skeletal progenitors accompanied with changes in global and regional DNA methylation. In a complementary fashion, knockdown of these genes stimulated chondrogenesis and inhibited cell death and senescence. We identified Sox9, Scleraxis, Bak1, and p21 as potential transcriptional targets responsible for its function in the developing digit model.

\section{Materials and methods}

We employed Rhode Island chicken embryos from day 4 to day 8.5 of incubation (id) equivalent to stages 23-34 $\mathrm{HH}$, and C57BL6 mouse embryos ranging from 12 to 14.5 days post coitum (pc).

\section{In situ hybridization and analysis of cell proliferation}

In situ hybridization of PFA-fixed limb specimens was performed in whole mount or $100-\mu \mathrm{m}$ vibratome sections. The samples were treated with $10 \mu \mathrm{g} / \mathrm{ml}$ of proteinase $\mathrm{K}$ for $20-30 \mathrm{~min}$ at $20^{\circ} \mathrm{C}$. Hybridization with digoxigeninlabeled antisense RNA probes was performed at $68^{\circ} \mathrm{C}$. Alkaline phosphatase-conjugated antidigoxigenin antibody (dilution 1:2000) was used (Roche). Reactions were developed with BM Purple AP Substrate precipitation (Roche).

The probes for Uhrf1 and Uhrf2 were obtained by PCR from RNA extracted from chick or mouse limb buds at initial stages of digit formation. Specific primers for chick Uhrf1 were: $5^{\prime}$-tccacatctattgcctcaacc- $3^{\prime}$ and $5^{\prime}$-gaacaccagattcgctcacc- $3^{\prime}$; for chick Uhrf2 $5^{\prime}$-agagttcaggtgagcgaagc$3^{\prime}$ and $5^{\prime}$-aggctcaacgtcatctctcc- 3 and for mouse Uhrf1: $5^{\prime}$ tgactctggctatggtgtgg- $3^{\prime}$ and $5^{\prime}$-gcctgatgttgccgtatagc- $3^{\prime}$; and for mouse Uhrf2 $5^{\prime}$-agagttcaggtgagcgaagc- $3^{\prime}$ and $5^{\prime}-$ tcgttcgattccttctgagg- $3^{\prime}$.

The distribution of proliferating cells in the autopod was analyzed in paraffin-embedded tissue sections by detection of bromodeoxyuridine (BrdU) incorporation $60 \mathrm{~min}$ after injection into the amniotic sac of $100 \mu \mathrm{l}$ of BrdU solution $(100 \mathrm{mg} / \mathrm{ml})$.

\section{Cell senescence, neutral red vital staining, TUNEL assay, and immunofluorescence}

The $\beta$-galactosidase activity assay ${ }^{20}$ was performed at pH 6 in vibratome sections of limb autopods fixed in $4 \%$ glutaraldehyde.

Neutral red staining, TUNEL assay, and electron microscopy were performed as described previously ${ }^{2}$.

Immunolabeling was performed in limb tissue samples fixed in $4 \%$ PFA. We employed both squashed interdigital tissue fragments or vibratome sections permeabilized with Triton X-100 in PBS. The following antibodies were employed: rabbit monoclonal anti-UHRF1 (DSG8E, Cell Signaling), rabbit polyclonal anti-UHRF2 (TA337863, OriGene); mouse monoclonal anti-UHRF2 (sc-398953, Santa Cruz Biotechnology); mouse monoclonal anti-5- 
methylcytosine (5-mC; 33D3, Eurogentech); rabbit polyclonal anti-SOX9 (AB5535,Milipore); mouse monoclonal anti- $\gamma$ H2AX (JBW301, Milipore-Upstate); and mouse monoclonal anti-BdrU (BU-33, Sigma Aldrich). Counterstaining to distinguish nucleus and cytoplasm was performed using fluorescent-phalloidin (Sigma Aldrich) or DAPI (Vector Laboratories). Observation were made with a LSM51O laser confocal microscope (Zeiss).

\section{In vivo treatments}

The transcriptional effects of FGF signaling in the expression of Uhrf genes were studied by analyzing the effects of the local administration of FGF2 (diluted at $0.5 \mathrm{mg} / \mathrm{ml}$, Peprotech), and the FGF inhibitor SU5402 (diluted at $4 \mathrm{mg} / \mathrm{ml}$, Calbiochem), using heparin acrylic (Sigma) or ion exchange (AG1-X2, Bio-Rad) microbeads as described $^{21}$.

\section{Mesodermal cultures}

Dissociated undifferentiated mesoderm from chick leg autopods at 4.5 i.d. $(25 \mathrm{HH})$ were cultured as micromasses containing a density of $2.0 \times 10^{7} \mathrm{cells} / \mathrm{ml}$. The chondrogenic outcome was studied under the microscope after Alcian blue staining ( $0.5 \%$ Alcian blue, at $\mathrm{pH} 1.0)$. Chondrogenesis was further quantified by the detection of Alcian blue dye extracted in $6 \mathrm{M}$ guanidine- $\mathrm{HCl}$ ( $\mathrm{pH} 5.8$ ), and the optical density was measured at $600 \mathrm{~nm}$.

\section{Cell nucleofection and targeted gene silencing}

Functional studies were performed by gain-of-function and loss-of-function approaches.

For gain-of-function experiments, skeletal progenitors were electroporated with constructs of chicken Uhrf1 (cUhrf1; OGa47434) or Uhrf2 genes (cUhrf2; OGa21255) cloned into the pcDNA3.1 vector (GenScript) employing the Multiporator System (Eppendorf) and cultured under high-density conditions as indicated above. For loss-offunction experiments, skeletal progenitors were electroporated with a short hairpin RNAi against Uhrf1 (sh-Uhrf1) or Uhrf2 (sh-Uhrf2) cloned into the pcU6-1-shRNA (a generous gift from Dr. Tim J. Doran). Transfections with the respective empty plasmids were employed as controls. After $48 \mathrm{~h}$ of culture, the level of gene regulation was confirmed by q-PCT and/or Western blot analysis.

\section{Evaluation of DNA methylation}

Changes in global methylation after Uhrf functional experiments were determined by the ELISA-based commercial kit Imprint ${ }^{\oplus}$ Methylated DNA Quantification (MDQ1; Sigma-Aldrich, St. Louis, MO, USA). One hundred and fifty nanograms of genomic DNA from our samples were incubated with capture and detection antibodies and their absorbance was measured at $450 \mathrm{~nm}$. The amount of methylated DNA present in the samples is proportional to the absorbance measured. Quantification of global DNA methylation was performed calculating methylation levels relative to the methylated control DNA $(50 \mathrm{ng} / \mu \mathrm{l})$ using the formula: [(A450 av sample-A450 av blank $) /(\mathrm{A} 450$ av methylated control DNA-A450 av blank) $] \times 100$.

We next selected Bak1 as potential target of Uhrf genes in the control of cell death. Changes in the methylation status of its promoter were studied by methylation sensitive restriction enzyme and quantitative polymerase chain reaction (MSRE-qPCR). Primers were designed using Primer3Plus online software. The selected PCR primer pair flanked the region of interest (based on the presence of informative restriction sites) within the promoter of Bak1: Fwd, AGCTGCAGCCTTCCCAGA; Rev, CTCTAGAGGCGCCTTGCAC. Genomic DNA samples were digested with a CpG-methylation-sensitive restriction enzyme (TauI $(3 \mathrm{U} / \mu \mathrm{l})$; ER1651 ThermoFisher Scientific) or with a non-CpG-methylation-sensitive enzyme (SacI $(10 \mathrm{U} / \mu \mathrm{l})$; ER1132 ThermoFischer Scientific) for $2 \mathrm{~h}$ according to the manufacturer's suggested temperature. SYBRGreen-based qPCR was carried out in triplicates with a total volume of $20 \mu \mathrm{l}$ per tube containing $1 \mu \mathrm{l}$ of genomic DNA (TauI-digested, SacI-digested, or undigested DNA), $0.4 \mu \mathrm{l}$ of each primer, $10 \mu \mathrm{l}$ of SYBR Select Master Mix (Life Technologies), and $8.2 \mu$ l of $\mathrm{H}_{2} \mathrm{O}$. Reactions were carried out in a StepOne Real Time System and analyzed by StepOne software v2.3 (Life Technologies). The relative percentage of methylated DNA was calculated according to the equation $2^{-\Delta \Delta C t}$ employing as normalizers the $\mathrm{Ct}$ values of both SacI digested and undigested samples ${ }^{22}$.

\section{Western blot analysis}

The total amount of UHRF proteins in control and treated tissue samples was evaluated by Western blot analysis. Total proteins were extracted by lysis from interdigits or dissociated micromass cultures. After determining the protein concentration, $30 \mu \mathrm{g}$ of each sample was loaded onto a $12.5 \%$ SDS polyacrylamide gel, electrophoresed and transferred to PVDF membranes. The membranes were incubated with primary antibodies (see "Immunofluorescence" section). Protein bands were detected with an Odyssey ${ }^{\mathrm{TM}}$ Infrared-Imaging System (LiCor Biosciences). Immunoblots were developed with antimouse IRDye800DX or anti-rabbit IRDye680DX as secondary antibodies (Rockland Immunochemicals, USA).

\section{Flow cytometry}

Control, Uhrf1 or Uhrf2 gene-overexpressing, or Uhrf1 or Uhrf2 gene-silenced cultures were dissociated. One million cells were used in each test. For propidium iodide (PI) staining, the cells were washed with PBS and fixed in $90 \%$ ethanol. The samples were incubated overnight at 
$4{ }^{\circ} \mathrm{C}$ with $0.1 \%$ sodium citrate, $0.01 \%$ Triton $\mathrm{X}-100$, and $0.1 \mathrm{mg} / \mathrm{ml}$ PI. The cell suspension was subjected to flow cytometry analysis in a Cytoflex (Beckman Coulter) and analyzed with the Cytexpert software.

\section{Real-time quantitative PCR (q-PCR) for gene expression analysis}

Total RNA was extracted using the NucleoSpin RNA kit (Macherey-Nagel). First-strand cDNA was synthesized using random hexamers and the High Capacity cDNA Reverse Transcription Kit (Life Technologies). The cDNA concentration was adjusted to $0.5 \mu \mathrm{g} / \mu \mathrm{l}$. SYBRGreen (Life Technologies)-based q-PCR was performed using the Mx3005P system (Stratagene). Rpl13 was chosen as the normalizer in interdigital samples and Gapdh in cultures. Mean values for fold changes were calculated. Expression level was evaluated relative to a calibrator according to the $2^{-(\Delta \Delta \mathrm{Ct})}$ equation. Each value represents the mean \pm SEM of at least four independent samples obtained under the same conditions. Data were analyzed using Student's $t$ test or ANOVA followed by Bonferroni test for post-hoc comparisons. Statistical significance was set at $p<0.05$. qPCR-specific primers analyzed in this study would be provided upon request.

\section{Results}

Interdigital and interphalangeal joint expression domains of UHRF1 and UHRF2 in the embryonic limb

A preliminary search for the interdigital expression of epigenetic regulators revealed high expression levels of Uhrf1 and Uhrf2 genes compared with interdigital gene markers $^{23}$ (Supplementary Fig. 1). Therefore, we generated probes to explore their expression by in situ hybridization. As shown in Fig. 1a-j, Uhrf genes show similar expression pattern in the interdigital mesoderm, preceding (id 5-6) and during the whole remodeling period (id 6.5-8; Fig. 1a-j). This period is characterized by proliferation arrest ${ }^{24,25}$ (Fig. $1 \mathrm{~m}$ ), and degeneration detectable by $\beta$-galactosidase labeling (Fig. 10), neutral red vital staining (Fig. 1p), $\gamma \mathrm{H} 2 \mathrm{AX}$ immunolabeling (DNA damage marker; Fig. 1q), and TEM (Fig. 1r). Analysis of the gene expression in tissue sections (Fig. 1e, j) detected additional domains in the developing interphalangeal joints that mark a region where cells also undergo programmed cell death and senescence (Fig. 1o). Quantification of interdigital gene expression showed that Uhrf1, after a short downregulation preceding id 6 , maintained in subsequent stages expression levels higher than other interdigital tissue markers (Fig. 1k, see also Supplementary Fig. 1). In contrast, the expression level of Uhrf2 remained relatively constant throughout the course of tissue remodeling (Fig. 11). Western blot analysis confirmed the presence of high levels of UHRF1 and UHRF2 proteins through the course of interdigit remodeling (Fig. 1n).
A similar expression pattern for both genes was observed in mouse embryos. As shown in Fig. 2, by day 12 pc, Uhrf genes are expressed in the undifferentiated autopodial mesoderm and in subsequent stages, expression becomes restricted to the interdigital mesoderm. By day $14 \mathrm{pc}$, interdigit regression is almost accomplished and Uhrf gene expression become restricted to the zones of joint formation (Fig. 2d, h)

\section{Cellular distribution of UHRF in the interdigital mesoderm}

The protein distribution was analyzed by immunofluorescence in mouse and chick interdigits (Figs. 3 and 4). Both proteins showed a diffuse pattern throughout the nucleus with distinctive foci of labeling intensification (Figs. 3a and 4a). Moderate cytoplasmic immunolabeling was also observed (Fig. 4a). The association of UHRF proteins with epigenetic markers and degenerative events was next explored (Figs. $3 \mathrm{~b}-\mathrm{d}$ and $4 \mathrm{~b}-\mathrm{d}$ ). We first analyzed the relation between UHRF proteins and methylated DNA monitored with 5-mC immunolabeling. In chick cells, 5-mC immunolabeling appeared as dots of variable size (Fig. $3 b^{\prime}$ and $c^{\prime}$, and $4 c^{\prime}$ ). In mouse cells, 5-mC labeling appeared as characteristic rings (Fig. 4b'). In both species UHRF1 and UHRF2 spots were often associated with 5-mC-positive marks. Of note, UHRF proteins often occupied the core of the 5-mC rings in mouse cells (inset of Fig. 3b" , and Fig. $4 \mathrm{~b}^{\prime \prime}$ ). Colocalization of UHRF proteins with $5-\mathrm{mC}$ foci were appreciated for UHRF1 (Fig. $3 \mathrm{~b})$. This overlapping expression is consistent with the demonstrated role of UHRF1 in recruiting DNA methyl transferase 1 and histone deacetylase 1 to chromatin regions containing $\mathrm{CpG}$ dinucleotides. Of relevance, immunolabeling for both UHRF1 and 2 appeared intensified at the initial stages of the dying process (Figs. 3c and 4c). These initial stages of cell degeneration are identified by a progressive loss and peripheral displacement of $5-\mathrm{mC}$ labeling. In contrast, at stages of overt apoptosis UHRF positivity was lost (Figs. 3d and 4d).

\section{Regulation of Uhrf gene expression by FGF signaling}

The growth and differentiation of the limb skeletal progenitors is controlled by FGFs. FGFs maintain proliferation and delay cell death and chondrogenic differentiation $^{21,26-28}$. The degeneration of the interdigital tissue and the completion of digit formation are associated with the extinction of FGF signaling. Therefore, we investigate the influence of FGFs on the expression of UHRF genes. As shown in Fig. 5a-f, the expression levels of FGF8 and FGF10, which are the most characteristic FGF genes expressed in the autopod at the studied stages, become downregulated at id 6 (Fig. 5a-f). As described above (Fig. 1k), id 6 is the period that marks the decreased expression of Uhrf1. In subsequent stages, when downregulation of FGFs is intensified, both Uhrf1 and Uhrf2 

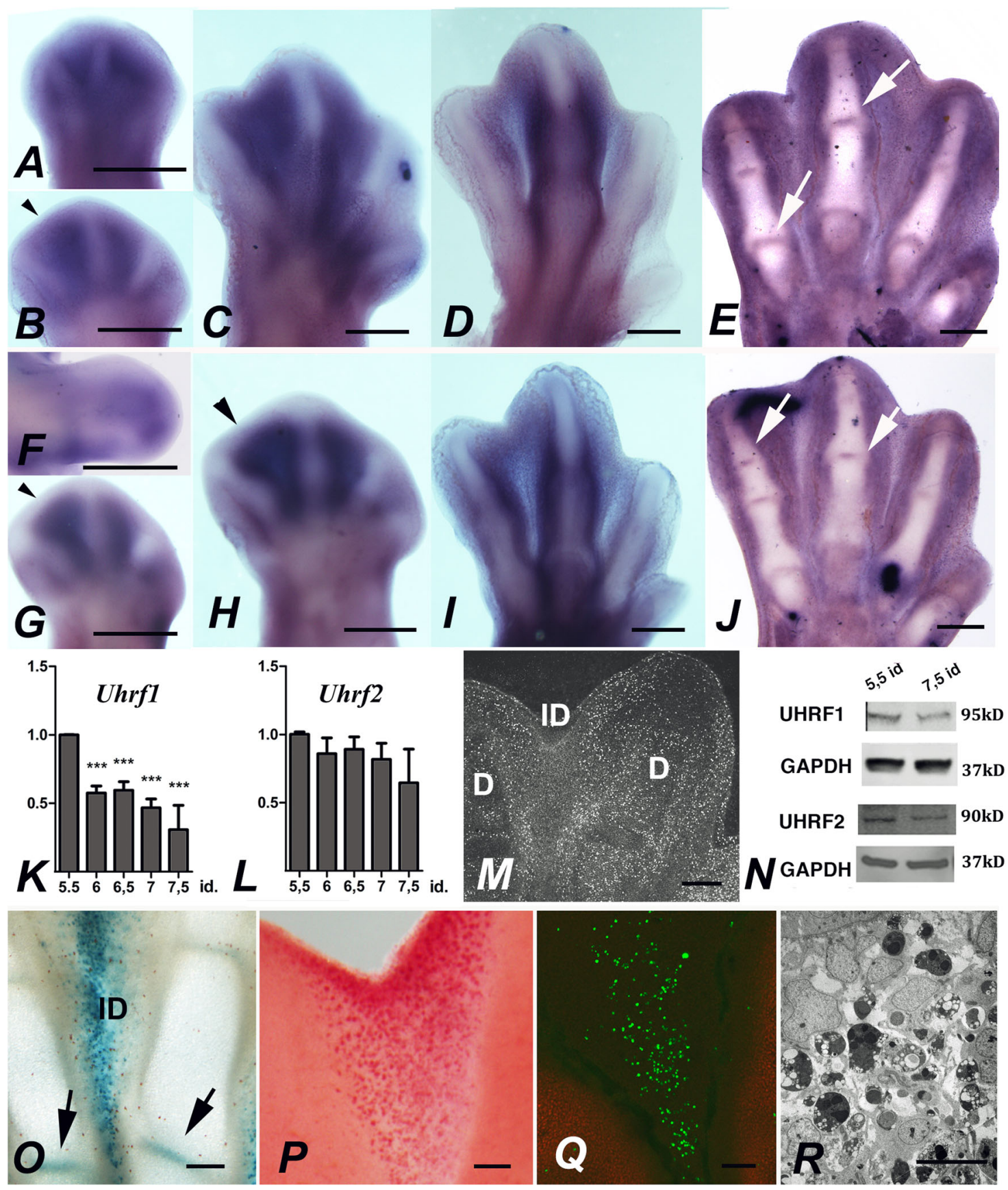

Fig. 1 Uhrf genes are expressed in the remodeling interdigits. Expression of Uhrfl (a-e) and Uhrf2 (f-j) in the interdigital tissue and joint forming regions of chick leg buds.Whole mount in situ hybridizations at id 5 (a), 4.5 (f), 5.5 (b and $\mathbf{g}), 6.5$ (c, h), and 7.5 (d, i). Arrowheads in $\mathbf{g}$ and $\mathbf{h}$ show a characteristic absence of Uhrf2 transcripts in the subridge mesoderm. e and $\mathbf{j}$ are in situ hybridizations in vibratome tissue sections at id 7.5 to show the joint domains (arrows) of Uhrf1 (e), and Uhrf2 (j). $\mathbf{k}$ and $\mathbf{I}$ are charts showing the expression levels of Uhrf1 (k) and Uhrf2 (I) in the course of interdigit tissue remodeling, considering a value of 1 for id 5.5. Note the decreased expression of Uhrf1 between id 5.5 and 6. $\mathbf{m}$ BrdU incorporation to mark mitosis in the autopod at id 6 . Note the almost absence of cells labeled in the interdigital tissue (ID) compared with the digit regions (d). $\mathbf{n}$ Western blotting showing the UHRF1 and UHRF2 proteins in the interdigital tissue at id 5.5 and 7.5 the stage preceding and the peak of degeneration. o-r $\mathbf{r}$ illustrations of the most characteristic features of the third interdigit in autopods at the peak of degeneration (id 7.5). o Senescence-specific beta-galactosidase activity. Note positivity in the interdigit (ID) and in the developing joints (arrows); $\mathbf{p}$ interdigit after neutral red vital staining; q immunolabeling with anti- $-\mathrm{H} 2 \mathrm{AX}$ showing interdigital cells undergoing DNA damage. Digit rays are labeled red with anti-Sox9; $\mathbf{r}$ transmission electron microscopic image to show the abundance of dark apoptotic cells in the interdigital mesoderm. ${ }^{* *} p<0.001$. Bars $=200 \mu \mathrm{m}$ $(\mathbf{a}-\mathbf{j}, \mathbf{m}, \mathbf{o}-\mathbf{q}) . \operatorname{Bar}=15 \mu \mathrm{m}(\mathbf{r})$ 


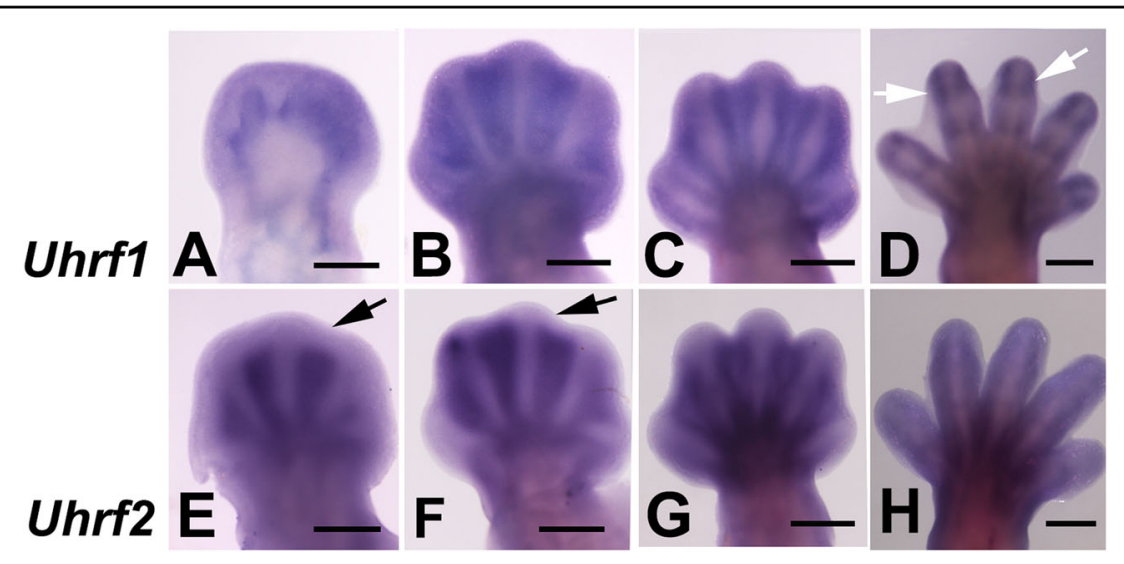

Fig. 2 In situ hybridizations showing the expression of Uhrf1 (a-d) and Uhrf2 (e-h) in the mouse autopod during interdigit remodeling. ad show the expression of Uhrf1 at pc days $12(\mathbf{a}), 13(\mathbf{b}), 13.5$ (c), and 14 (d). Note the presence of joint domains by pc 14 (arrows in $\mathbf{d})$. e-h show the expression of Uhrf2 at pc days $12.5(\mathbf{e}), 13(\mathbf{f}), 13.5(\mathbf{g})$, and $14(\mathbf{h})$. Note the fading of the interdigital domains in the distal subectodermal region (arrows in e and $\mathbf{f}$ ). Bars $=500 \mu \mathrm{m}$

remain expressed at high levels. Interdigital application of a FGF-bead moderately upregulated the expression of Uhrf1 (Fig. 5g, h and j), but not Uhrf2 (Fig. 5k-l and n). In contrast, the local application of the FGF inhibitor SU5402 downregulated both Uhrf genes (Fig. 5i, j, and m, n). Together, these findings suggest that FGF signaling promotes only the expression of Uhrf1 but sustains the expression of both Uhrf genes.

\section{Expression and functional analysis of Uhrf genes during chondrogenic differentiation}

The absence of Uhrf transcripts in the differentiating digit rays revealed these factors as potential inhibitors of chondrogenic differentiation. To test this hypothesis we monitored their expression in micromass cultures of limb skeletal progenitors (Fig. 6). This in vitro assay mimics the process of digit cartilage differentiation in vivo. The skeletal progenitors grow initially in an undifferentiated state, but by $48 \mathrm{~h}$ of culture, they aggregate to form chondrogenic nodules (Fig. 6a). The nodules are separated from each other by cells that retain morphological and transcriptional characteristics of fibrous connective tissue ${ }^{29}$. In subsequent days of culture, the chondrogenic nodules increased in number and size (Fig. $6 \mathrm{a}-\mathrm{C}$ ), forming by the end of the second week an almost continuous sheet of cartilage $^{30}$. Uhrf1 expression showed the highest levels on day 1 of culture, but the expression declined to half by day 2 , progressing to more moderate levels during subsequent days of culture (Fig. 6d). At difference of Uhrf1, Uhrf2 maintained uniform expression levels during the first 4 days of culture (Fig. 6e). These results are compatible with an antichondrogenic role, at least for Uhrfl.

To further explore their potential role in chondrogenesis, a functional analysis of $U h r f$ genes was performed by gain-of-function and loss-of-function experiments in the micromass assay (Fig. 6f-i). Chondrogenesis was significantly downregulated in 5-day cultures of progenitors transfected with either Uhrf1 (Fig. 6f) or Uhrf2 (Fig. 6h) genes. In a complementary fashion, chondrogenesis was significantly upregulated after the knockdown of either Uhrf1 (Fig. 6g) or Uhrf2 (Fig. 6i), via transfection of the corresponding sh-RNAi.

\section{Uhrf genes modulate cell death and cell cycle progression of skeletal progenitors}

From our previous experiments, we concluded that Uhrf genes inhibit mesenchymal precursor differentiation. As mentioned above, skeletal progenitors that do not differentiate during limb development, undergo senescence and cell death. Therefore, we next explored whether Uhrf genes influenced the degenerative fate of the excess progenitors. For this purpose, changes in cell proliferation and death were analyzed in the micromass culture assay. After 2 days of culture, cell death was significantly regulated in gain-of-function and loss-of-function experiments (Fig. 7a). Over-expression of Uhrf1 increased the number of dead cells more than three times, and its silencing decreased cell death by $25 \%$. In turn, the overexpression of Uhrf2 increased cell death by two-fold, and its knockdown decreased cell death by $35 \%$. Changes in the intensity of cell death were accompanied by cell cycle arrest in the S-phase (Fig. 7b, c). Hence, the rate of cells in the S phase increased by $150 \%$ after Uhrf1 overexpression and was reduced by $20 \%$ in loss-of-function experiments (Fig. 7b). The effect of Uhrf2 on cell cycle progression was less consistent. Uhrf2 overexpression increased the number of cells in S phase by $20 \%$ and silencing reduced S phase cells at a similar rate (Fig. 7c). Together, these findings are consistent with the arrest of cell cycle at $\mathrm{S}$ phase, as occurs in response to DNA damage. 

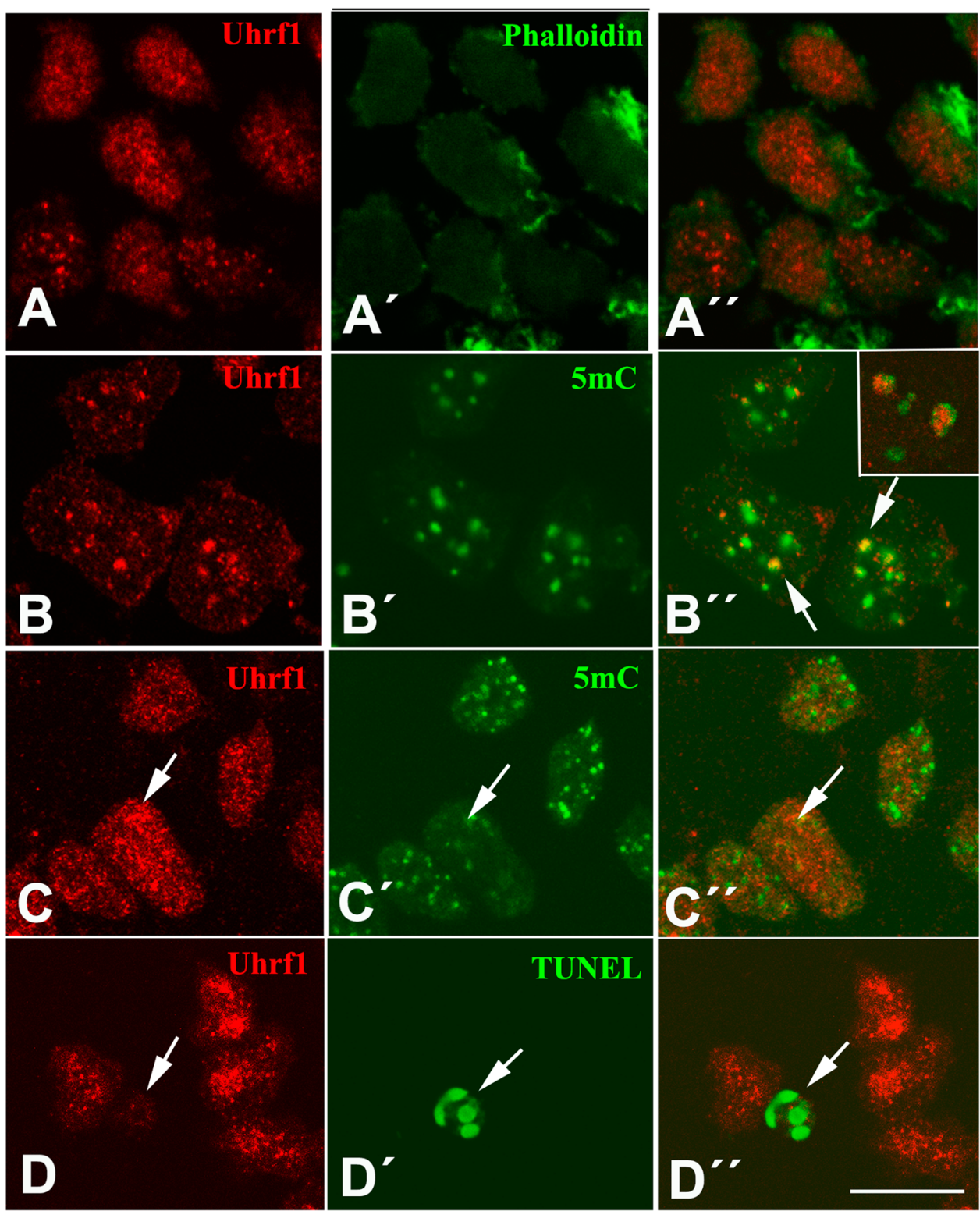

Fig. 3 UHRF1 immunolabeling (red) of chick interdigital cells at $\mathbf{7}$ id. a-a" UHRF1 (red) in combination with phalloidin (green) show the nuclear localization of UHRF1. (a) UHRF1 labeling, (a') phallodin labeling showing cytoplasmic actin; (a') merged images. (b-b") UHRF1 (red) in combination 5-mC immunolabeling (green) to show the overlapping distribution of UHRF1 in zones of DNA methylation (arrow). (b) UHRF1 labeling; (b') 5-mC immunolabeling; ( $\left.\mathbf{b}^{\prime \prime}\right)$ merged images; inset in this image shows the association between UHRF1 and 5-mC rings in mouse interdigital cells. (c-c') Double immunolabeling for UHRF1 (red) and 5-mC (green) to show the increased UHRF1 labeling in cells with reduced DNA methylation (arrows). (c) UHRF1 labeling; ( $\mathbf{c}^{\prime}$ ) 5-mC immunolabeling; ( $\mathbf{c}^{\prime \prime}$ ) merged image. (d-d') Immunolabeling of UHRF1 (red) in combination with TUNEL (green) to show the loss of UHRF1 in TUNEL-positive apoptotic cells (arrows). (d) UHRF1 labeling; (d') TUNEL labeling; (d') merged image. Bar = $10 \mu \mathrm{m}$

\section{Transcriptional influence of Uhrf genes}

UHRF factors act through the modulation of gene expression at the transcriptional level. Therefore, we next explored the influence of Uhrf genes in the transcription of a panel of genes associated with differentiation, proliferation, and apoptosis of limb mesenchymal progenitors (Table 1).
The dual differentiation of limb skeletal progenitors is regulated by a balance between Sox9, and Scleraxis, which are master chondrogenic and fibrogenic genes ${ }^{31,32}$, respectively. Both genes regulate the dichotomic differentiation of progenitors into cartilage or fibrous tissue. Increased chondrogenesis in knockdown experiments was preceded by downregulation of Scleraxis, while the 

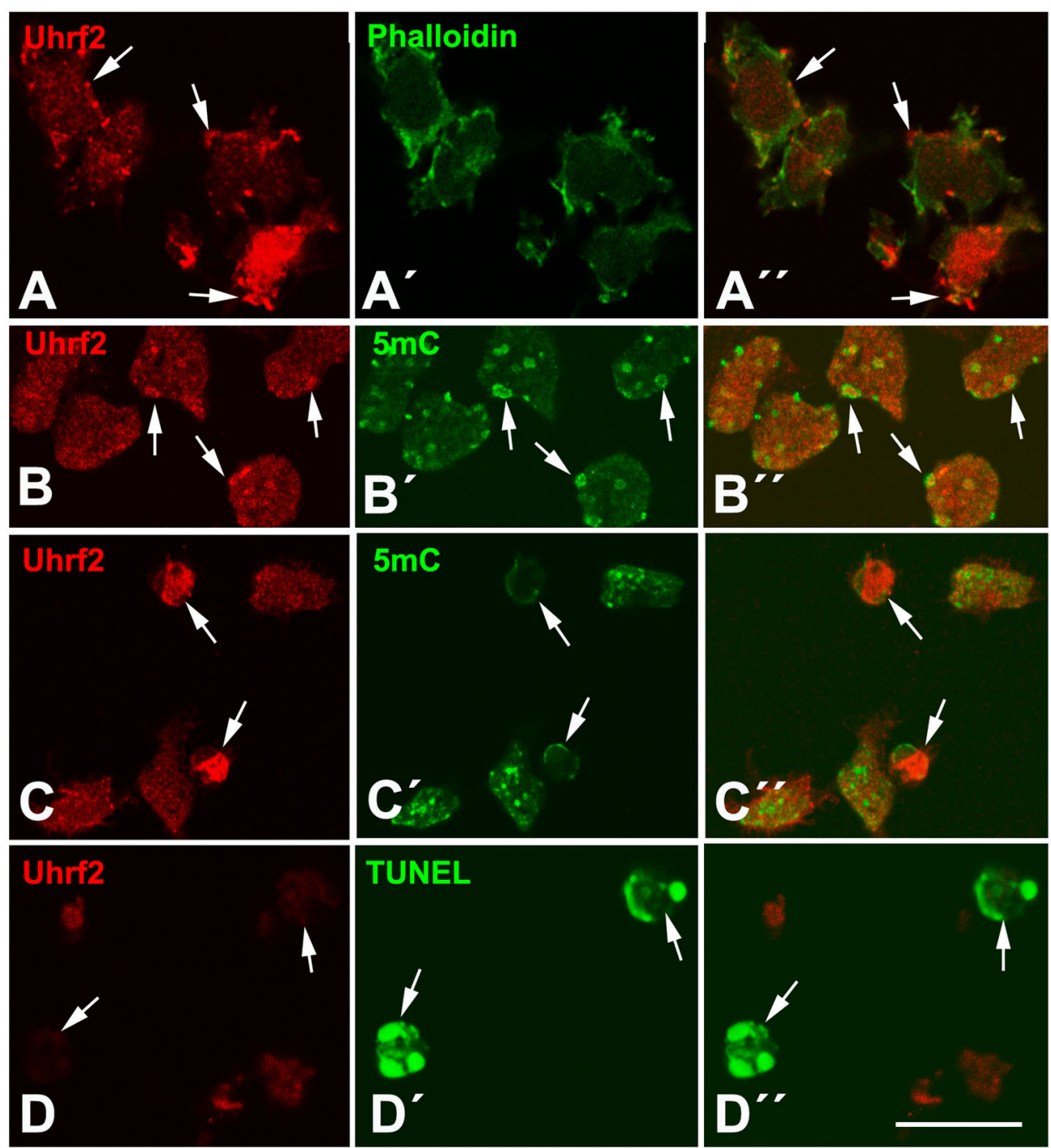

Fig. 4 UHRF2 immunolabeling of interdigital cells. UHRF2 immunolabeling (red) of chick (a, c, d) and mouse (b) interdigital cells.(a-a') UHRF2 (red) in combination with phalloidin (green) showing the occurrence of both nuclear and cytoplasmic (arrow) localization of UHRF2. (a) UHRF2 labeling, (a') phallodin labeling showing cytoplasmic actin; (a') merged images. (b-b') UHRF2(red) in combination 5-mC immunolabeling (green) to show the presence of UHRF2 positivity in the core of 5-mC rings (arrows). (b) UHRF2 labeling; (b') 5-mC immunolabeling; (b') merged images. (c-c') Double immunolabeling for UHRF1 (red) and 5-mC (green), to show the increased immunolabeling in cells with reduced DNA methylation (arrows). (c) UHRF2 labeling; (c') 5-mC immunolabeling; ( $\mathbf{c}^{\prime \prime}$ ) merged image. (d-d') Immunolabeling of UHRF1 (red) in combination with TUNEL (green) to show the loss of UHRF2 in apoptotic cells TUNEL-positive (arrows). (d) UHRF1 labeling; (d') TUNEL labeling; (d') merged image. Bar = $10 \mu \mathrm{m}$

upregulation of $\operatorname{Sox} 9$ was not detectable until the overt differentiation of the micromass cultures (day 4 of culture). In contrast, the downregulation of Sox 9 in experiments of gain-of-function experiments was more precocious than the changes in the expression of Scleraxis.

The expression analysis of members of the $B c l 2$ cell death gene family showed a significant regulation of $B a k 1$ in association with changes in the intensity of cell death. The overexpression of Uhrf1 increased Bak1 expression by $250 \%$, while gene silencing decreased Bak1 expression by half. Uhrf2 functional experiments caused a similar but more moderate regulation of Bak1. Other members of the $B c l 2$ gene family, were either not regulated or moderately regulated (Table 1).

To explore the influence of Uhrf genes in cell senescence we selected $p 21$ as the most conspicuous marker of embryonic developmental senescence. As shown in Table $1, p 21$ was upregulated six-fold after $U h r f 1$ overexpression and two-fold after Uhrf2 overexpression. In turn, $p 21$ was significantly downregulated after the knockdown of either Uhrf1 $(0.5 \times)$ or Uhrf2 $(0.3 \times)$. 


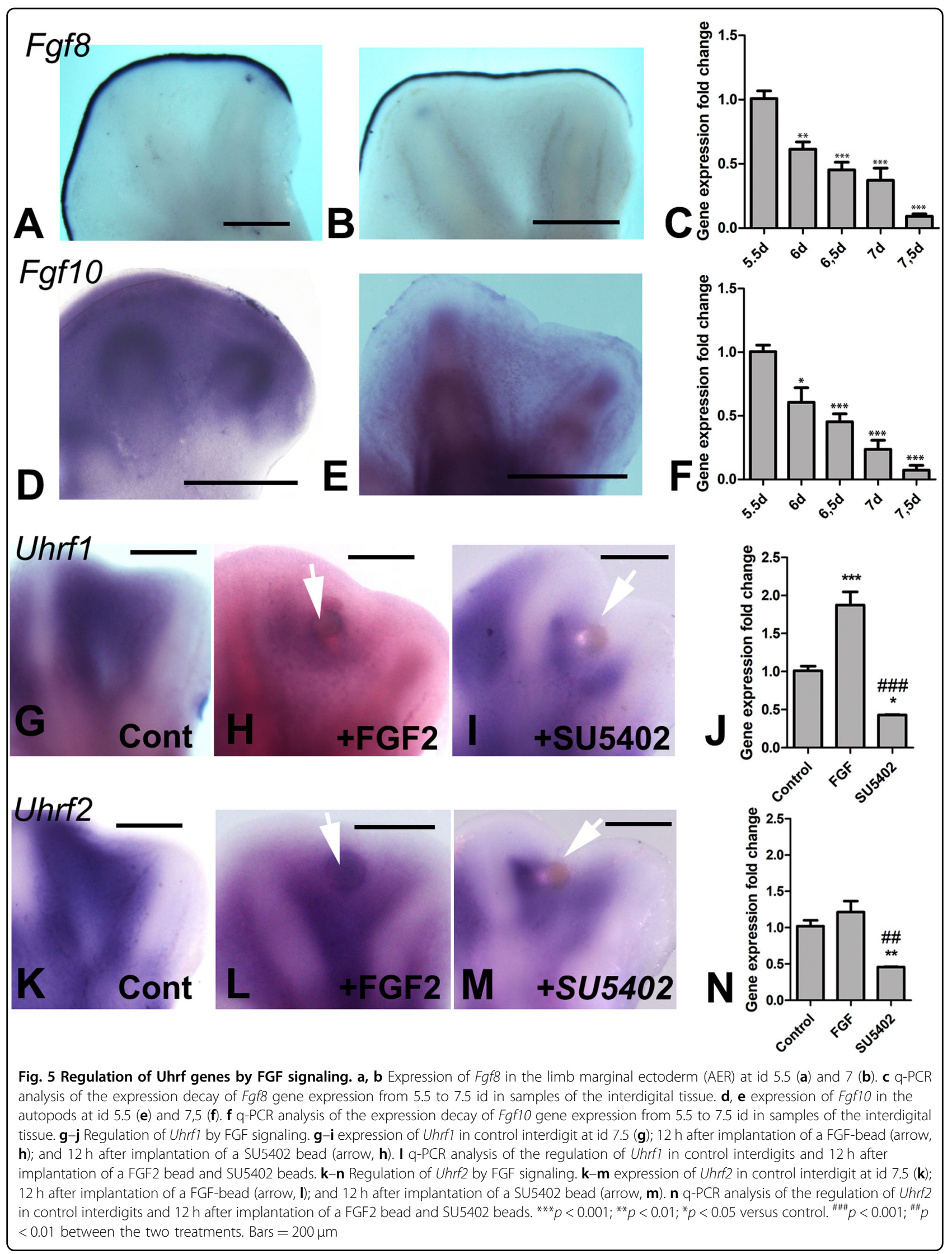




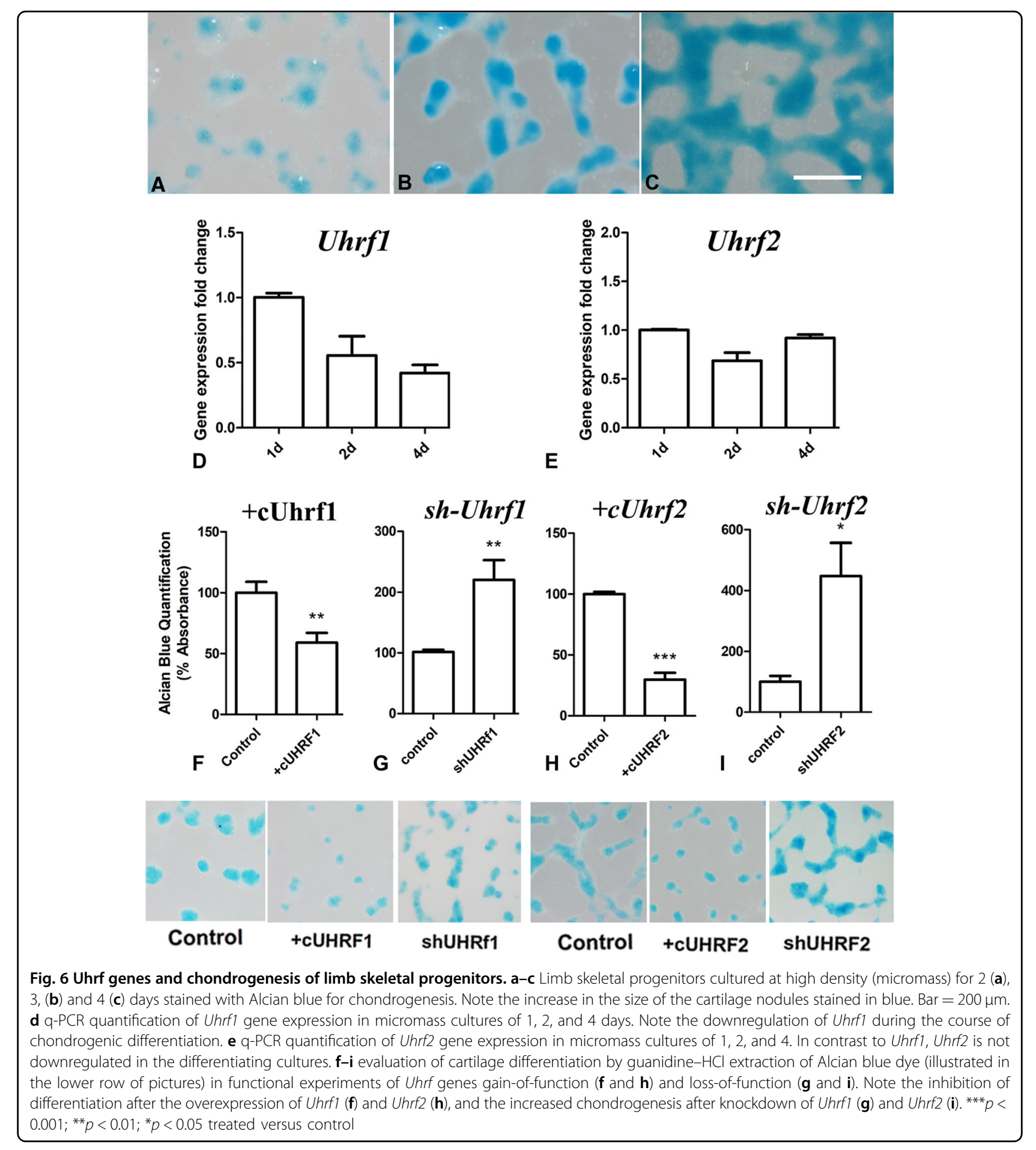

\section{Regulation of DNA methylation by Uhrf genes}

Considering the functional implication of UHRFs in genomic DNA methylation, we first analyzed changes in global methylation in micromass cultures subjected to Uhrf gene overexpression or silencing. Global methylation was significantly increased in 2-day cultures transfected with
Uhrf1 or Uhrf2 genes (Fig. 8a), but was not modified at statistical significant levels when progenitors were subjected to Uhrf's gene silencing. However, we detected a mild decrease in global methylation after Uhrf1 gene silencing.

To address the functional significance of methylation in the regulation of cell death by Uhrf genes, we select Bak1 


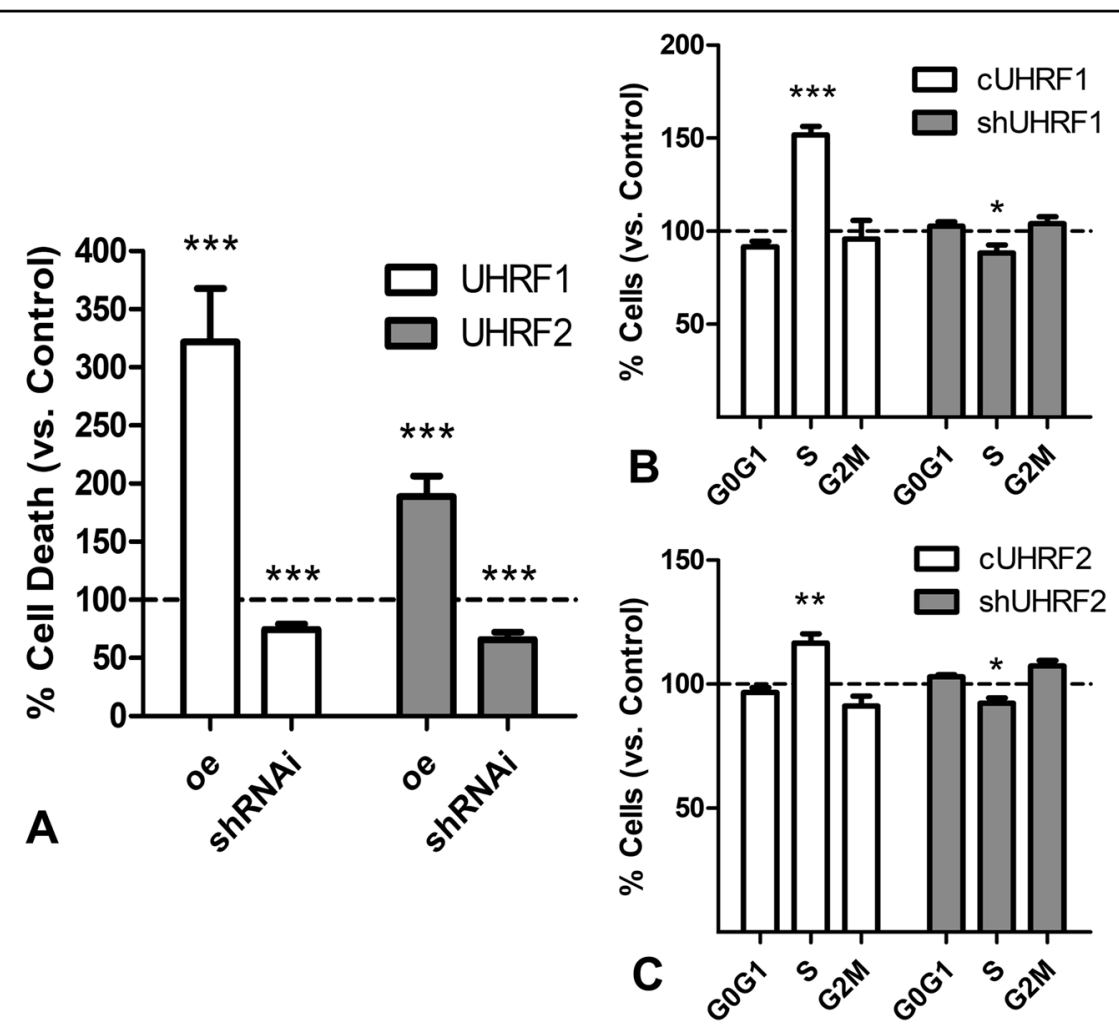

Fig. 7 Regulation of cell death and cell cycle by UHRFs. a Chart showing differences in the intensity of cell death evaluated by flow cytometry between control progenitors and progenitors overexpressing (oe) or subjected to silencing (shRNAi) of Uhrf1 (white columns) and Uhrf2 (gray columns).The level of cell death in control cultures was considered $100 \%$ and is represented by the dotted line. $\mathbf{b}$ Graphic illustrations comparing the proportion of cells at different cell cycle stages in cultures overexpressing Uhrfi (white columns) and after gene silencing (gray columns) versus control cultures (represented by the dotted line). c Graphic illustrations comparing the proportion of cells at different cell cycle stages in cultures overexpressing Uhrf2 (white columns) and after gene silencing (gray columns) versus control cultures (represented by the dotted line). ${ }^{* * *} p<0.001$; ${ }^{* *} p<0.01 ;{ }^{*} p<0.05$ treated versus control

to explore methylation changes in CpG islands of its promoter by MSRE-qPCR (Fig. 8b). Gain-of-function experiments of both Uhrf1 or Uhrf2 were followed by decreased methylation. The decrease was particularly intense after Uhrf1 overexpression, and more moderated for Uhrf2. As observed for global methylation, gene silencing did not change at significant levels methylation of Bak1 promoter.

\section{Functional redundancy of Uhrf genes}

We first analyzed the influence of Uhrf genes on the expression of each other. As shown in Table 1, neither overexpression nor gene silencing of the Uhrf1 gene regulated the expression of Uhrf2 at significant levels. However, overexpression and silencing of $U h r f 2$ were followed by a moderate regulation of Uhrf1.

We next analyzed by flow cytometry changes in cell death in combined transfections of overexpression plasmids and sh-RNA inhibitory constructs (Supplementary Fig. 2A, B). Transfection of Uhrf2 in combination with shRNAi-Uhrf1 neutralized the cell death promoting effect of Uhrf2 (Supplementary Fig. 2A). In these experiments, the intensity of cell death became similar to that observed after transfections with shRNAi-Uhrf1 only. In a similar fashion, the inhibitory influence on cell death of sh-RNAiUhrf2 was also abolished when it was co-transfected with Uhrf1 (Supplementary Fig. 2B). Finally, the increased cell death induced separately by Uhrf1 or Uhrf2 lacked statistically significant differences with the level of cell death induced by double transfections with both Uhrf genes (Supplementary Fig. 2C). Together, these findings suggest common and complementary roles for both genes in the embryonic limb mesoderm.

\section{Discussion}

Current knowledge of the role of UHRF proteins in developing vertebrates is scarce because mouse $\mathrm{KO}$ for Uhrf1 die early in gestation ${ }^{33}$, and mice deficient in Uhrf2 lack skeletal phenotype ${ }^{18}$. However, the implication of Uhrf1 in the formation and growth of various organs has been observed in mutant zebrafish embryos ${ }^{16}$, and the influence of Uhrf1 in cartilage maturation, has been 
demonstrated via a conditional knockout (Uhrf1-cKO) targeted to the limb mesoderm ${ }^{17}$. The skeletal phenotype of Uhrf1-cKO mice provided evidence for a role for UHRF1 in the elongation of appendicular bones during the postnatal period by regulating the growth and differentiation of the growth plates. Our study extends the function of these genes to the stages of digit

Table 1 Transcriptional analysis of limb progenitors subjected to gain-of-function and loss-of-function of either Uhrf1 or Uhrf2 genes

\begin{tabular}{|c|c|c|c|c|}
\hline & cUhrf1 & shUhrf1 & cUhrf2 & shUhrf2 \\
\hline \multicolumn{5}{|c|}{ Differentiation markers } \\
\hline$S c x$ & $1.02 \pm 0.11$ & $0.59 \pm 0.06^{* * *}$ & $1.17 \pm 0.20$ & $0.50 \pm 0.09^{* * *}$ \\
\hline Sox9 & $0.59 \pm 0.07^{* * *}$ & $0.93 \pm 0.09$ & $0.59 \pm 0.08^{* * *}$ & $0.80 \pm 0.15$ \\
\hline \multicolumn{5}{|c|}{ Senescence marker } \\
\hline p21 & $6.18 \pm 1.98^{*}$ & $0.56 \pm 0.07^{* * *}$ & $2.36 \pm 0.35^{* *}$ & $0.38 \pm 0.08^{* * *}$ \\
\hline \multicolumn{5}{|c|}{ Cell death markers } \\
\hline$B C l 2$ & $1.24 \pm 0.10$ & $1.12 \pm 0.06$ & $0.89 \pm 0.07$ & $1.01 \pm 0.14$ \\
\hline Bak1 & $2.44 \pm 0.51^{* *}$ & $0.59 \pm 0.03^{* * *}$ & $1.80 \pm 0.34^{*}$ & $0.80 \pm 0.10$ \\
\hline Bid & $1.45 \pm 0.21$ & $0.85 \pm 0.06$ & $1.24 \pm 0.15$ & $0.92 \pm 0.15$ \\
\hline Bim & $0.99 \pm 0.04$ & $1.24 \pm 0.12$ & $0.91 \pm 0.19$ & $0.98 \pm 0.12$ \\
\hline Bmf & $1.72 \pm 0.31^{*}$ & $0.94 \pm 0.08$ & $1.02 \pm 0.13$ & $0.89 \pm 0.14$ \\
\hline Uhrfi & $10.33 \pm 2.88^{* *}$ & $0.44 \pm 0.03^{* * *}$ & $1.98 \pm 0.35^{*}$ & $0.69 \pm 0.07^{* * *}$ \\
\hline Uhrf2 & $1.26 \pm 0.12$ & $0.95 \pm 0.05$ & $10.31 \pm 2.53^{* *}$ & $0.50 \pm 0.04^{* * *}$ \\
\hline
\end{tabular}

The panel of genes analyzed included markers for skeletogenic differentiation, senescence, and apoptosis

${ }^{* * *} p<0.001 ;{ }^{* *} p<0.01 ;{ }^{*} p<0.05$ treated versus control

Statistic significant values are highlighted in bold skeletogenesis. Our study showed that $U h r f$ genes inhibit the chondrogenesis of skeletal progenitors. This function contrasts with the requirement of UHRF1 for the differentiation of postnatal chondrocytes grown in micromass culture $^{17}$. These different effects emphasize the dependence of Uhrf genes on their functions of factors whose presence in the cells is related to the stage of differentiation. In the embryo, the expression domains of Uhrf1 and Uhrf2 specifically mark undifferentiated progenitors. Furthermore, in the case of Uhrf1, the level of expression in vitro becomes progressively reduced in parallel with the differentiation of the progenitors into chondrocytes. These observations support a role for Uhrf genes, especially Uhrf1, in the maintenance of progenitors in an undifferentiated state.

We identified Sox9 and Scleraxis, as potential mediators for the antichondrogenic influence of UHRFs on the skeletal progenitors. Sox9 belongs to the high-mobility group of chromatin regulators and plays a central role in the differentiation of skeletal progenitors ${ }^{34-36}$. Scleraxis, is a basic helix-loop-helix transcription factor structurally associated with Sox9 and is involved in the formation of fibrous connective tissues ${ }^{31,35}$. Sox 9 promotes the onset of chondrogenic differentiation in the developing limb and its silencing leads to cell death of skeletal progenitors ${ }^{34,37}$. The function of SOX9 is modulated by epigenetic mechanisms via histone acetylation on chromatin ${ }^{35}$. Remarkably, in our study, the chondrogenic inhibition and increased cell death in Uhrf gene gain-of-function experiments involved a precocious downregulation of Sox9. In contrast, increased chondrogenesis in loss-offunction experiments were preceded by downregulation of Scleraxis. This finding suggests a double and complementary function of Uhrf genes via the activation or
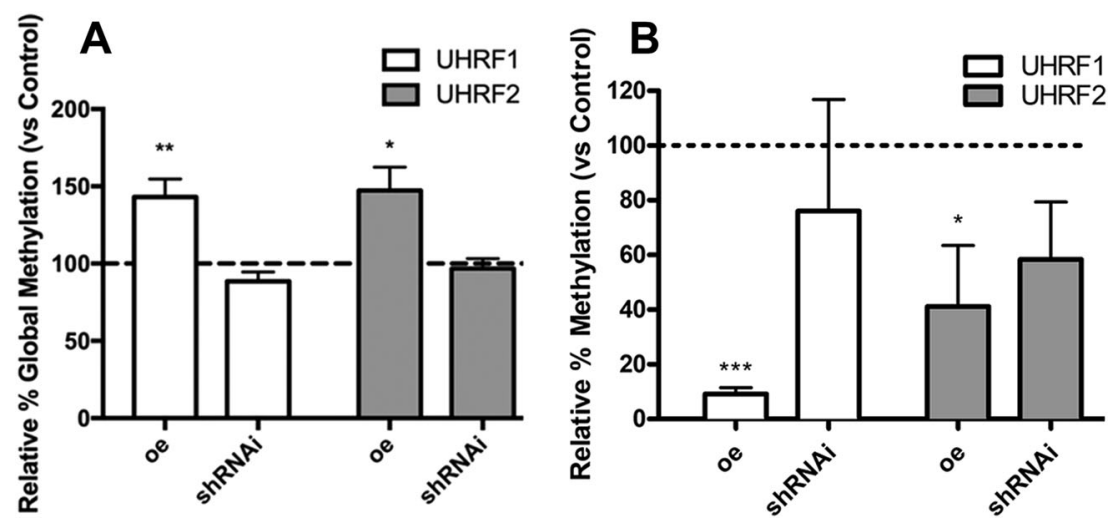

Fig. 8 Changes in DNA methylation by UHRFs. a Chart showing differences in global methylation evaluated by ELISA between control progenitors and progenitors overexpressing (oe) or subjected to silencing (shRNAi) of Uhrf1 (white columns) and Uhrf2 (gray columns).The level of global methylation in control cultures was considered 100\% and is represented by the dotted line. b Methylation level of CpG islands in the Bak1 promoter evaluated by MSRE-qPCR between control progenitors and progenitors overexpressing (oe) or subjected to silencing (shRNAi) of Uhrf1 (white columns) and Uhrf2 (gray columns). The level of CpG methylation in control cultures was considered 100\% and is represented by the dotted line. ${ }^{* * *} p<0.001 ;{ }^{* *} p<0.01 ;{ }^{*} p<0.05$ treated versus control 
repression of the promoters of master genes that establish the fate of the skeletal progenitors.

UHRF proteins play pivotal functions in carcinogenesis modulating DNA methylation and the histone functional code of tumor cells ${ }^{38-40}$. Consistent with these facts, here we show that both $U h r f$ genes promote global methylation in skeletal progenitors. Changes induced in cancer cells by dysregulation of Uhrf genes include cell proliferation, cell senescence, apoptosis, increased metastatic potential, and increased sensitivity to DNA-damaging agents ${ }^{14,15,40-44}$. In most cases the function of UHRF proteins takes place in coordination with other chromatin-modifying proteins ${ }^{44}$. This feature explains that the function of UHRF proteins varies in a cell cycle-dependent and cell lineagedependent manner.

The degenerative events accounting for interdigit remodeling occur in a sequential fashion. In the $12-24 \mathrm{~h}$ preceding the onset of massive apoptosis, the interdigital cells undergo proliferation arrest ${ }^{24}$, and intense DNA damage ${ }^{5}$. These changes are next followed by senescence ${ }^{4}$ and massive apoptosis to accomplish interdigit removal. Between id 5.5 and id 6, we observed a partial decrease in the interdigital expression of Uhrf1 that correlated with the downregulation of FGF genes at the end of limb morphogenesis. In subsequent stages of degeneration (from id 6.5 to id 8 ) both UHRF1 and UHRF2 maintained elevated protein and transcriptional expression levels, suggesting an active participation of both UHRF proteins in tissue regression.

Our functional approaches established the implication of both Uhrf genes in the regulation of the cell death of skeletal progenitors, and in the expression of $p 21$, a gene that plays a pivotal role in embryonic developmental senescence ${ }^{4}$. Additionally, the overexpression of Uhrf genes arrested the cell cycle in $\mathrm{S}$ phase, a characteristic feature of senescent cells associated with DNA damage. Cell death appeared significantly upregulated in progenitors overexpressing either Uhrf1 or Uhrf2, and significantly reduced in loss-of-function experiments of either gene. UHRFs have been implicated in the regulation of apoptosis in tumoral systems by distinct mechanisms, including the transcriptional regulation of tumor suppressor genes ${ }^{38}$, selective regulation of proapoptotic genes ${ }^{14,45}$, the induction of global hypomethylation leading to changes in tumor radiosensitivity ${ }^{39}$, or by reducing the capacity to repair DNA damage ${ }^{46}$. Remarkably, in our experimental system the intensification and attenuation of cell death correlated with changes in the expression of $B a k 1$, a characteristic proapoptotic member of the $B c l 2$ gene family with a demonstrated role in the regression of the interdigits ${ }^{47}$. Furthermore, up-regulation of Bak1 was associated with hypomethylation of the CpG islands of its promoter.

In normal cells, UHRF1 participates in the regulation of the cell cycle, and in cooperation with the DNA methyl transferase 1 (DNMT1), UHRF1 maintains the DNA methylation status of dividing cells. It has been proposed that Uhrf1 plays a primary role in cell senescence regulating the expression DNTM $1^{48}$. In our system, DNMT1 was not regulated by Uhrf1 or Uhrf2 (unpublished observation), but, in turn, we detected an intense regulation of the cell senescence master gene $p 21$ indicative of the direct implication of these genes in cell senescence. A similar regulation of $p 21$ by $U h r f 1$ has been reported in a number of systems ${ }^{44,49}$. However, in some tumoral cell lineages, UHRF1 and UHRF2 exerted negative transcriptional influence on the expression of $p 21^{50}$. This discrepancy is explained by the importance of specific cofactors that, together with UHRFs, form heteromeric binding complexes in the promotors of target genes, modulating their functions in a cell-cycle and cell-lineagedependent manner ${ }^{44}$.

In conclusion, our study uncovers a new level of regulation of interdigital apoptosis and cell senescence upstream of the components of the intrinsic pathway responsible for executing cell death in embryonic systems. Furthermore, our findings indicate that the balance between cell differentiation and cell stemness may be a central step in the initiation of the so-called "programmed cell death" associated with embryonic morphogenesis. The epigenetic functional profile of Uhrf genes in most studied systems, together with the changes in DNA methylation observed in our functional experiments, suggests that the structural organization of the chromatin may be a critical factor in the regulation of embryonic cell death and cell senescence.

\section{Acknowledgements}

We thank Montse Fernandez Calderon, Susana Dawalibi, and Sonia Perez Mantecon, for excellent technical assistance. This work was supported by a Grant (BFU2017-84046-P) from the Spanish Science and Innovation Ministry to J.A.M.

Conflict of interest

The authors declare that they have no conflict of interest.

\section{Publisher's note}

Springer Nature remains neutral with regard to jurisdictional claims in published maps and institutional affiliations.

Supplementary information accompanies this paper at (https://doi.org/ 10.1038/s41419-019-1575-4).

Received: 18 December 2018 Revised: 26 March 2019 Accepted: 4 April 2019

Published online: 25 April 2019

\section{References}

1. Hurle, J. M. \& Gañan, Y. Formation of extra-digits induced by surgical removal of the apical ectodermal ridge of the chick-embryo leg bud in the stages previous to the onset of interdigital cell-death. Anat. Embryol. 176, 393-399 (1987). 
2. Zuzarte-Luis, V., Montero, J. A., Kawakami, Y., Izpisua-Belmonte, J. C. \& Hurle, J. M. Lysosomal cathepsins in embryonic programmed cell death. Dev. Biol. 301, 205-217 (2007).

3. Arakawa, S. et al. Role of Atg5-dependent cell death in the embryonic development of Bax/Bak double-knockout mice. Cell Death Differ. 24, 1598-1608 (2017)

4. Lorda-Diez, C. I. et al. Apoptosis during embryonic tissue remodeling is accompanied by cell senescence. Aging 7, 974-985 (2015).

5. Montero, J. A., Sanchez-Fernandez, C., Lorda-Diez, C. I., Garcia-Porrero, J. A. \& Hurle, J. M. DNA damage precedes apoptosis during the regression of the interdigital tissue in vertebrate embryos. Sci. Rep. 6, 35478 (2016).

6. Zuzarte-Luís, V. et al. A new role for BMP5 during limb development acting through the synergic activation of Smad and MAPK pathways. Dev. Biol. 272 39-52 (2004).

7. Nebbioso, A., Tambaro, F. P., Dell'Aversana, C. \& Altucci, L. Cancer epigenetics: moving forward. PLoS Genet. 14, e1007362 (2018).

8. Liu, X. et al. Self-inflicted DNA double-strand breaks sustain tumorigenicity and stemness of cancer cells. Cell Res. 27, 764-783 (2017).

9. Palla, V. V. et al. Gamma-H2AX: can it be established as a classical cancer prognostic factor? Tumour Biol. 39, 1010428317695931 (2017).

10. Bronner, $C$. et al. The UHRF family: oncogenes that are drugable targets for cancer therapy in the near future? Pharmacol. Ther. 115, 419-434 (2007).

11. Un, F. et al. Modulating ICBP90 to suppress human ribonucleotide reductase M2 induction restores sensitivity to hydroxyurea cytotoxicity. Anticancer Res. 26, 2761-2767 (2006).

12. Mistry, $H$. et al. UHRF1 is a genome caretaker that facilitates the DNA damage response to gamma-irradiation. Genome Integr. 1, 7 (2010).

13. Iwata, A. et al. Intranuclear degradation of polyglutamine aggregates by the ubiquitin-proteasome system. J. Biol. Chem. 284, 9796-9803 (2009).

14. Lu, H. \& Hallstrom, T. C. The nuclear protein UHRF2 is a direct target of the transcription factor E2F1 in the induction of apoptosis. J. Biol. Chem. 288 23833-23843 (2013)

15. Muto, M. et al. Targeted disruption of Np95 gene renders murine embryonic stem cells hypersensitive to DNA damaging agents and DNA replication blocks. J. Biol. Chem. 277, 34549-34555 (2002)

16. Jacob, V. et al. DNA hypomethylation induces a DNA replication-associated cell cycle arrest to block hepatic outgrowth in uhrf1 mutant zebrafish embryos. Development 142, 510-521 (2015).

17. Yamashita, M. et al. Uhrf1 is indispensable for normal limb growth by regulating chondrocyte differentiation through specific gene expression. Development 145, dev157412 (2018).

18. Chen, $\mathrm{R}$. et al. The 5 -Hydroxymethylcytosine $(5 \mathrm{hmC})$ reader UHRF2 is required for normal levels of $5 \mathrm{hmC}$ in mouse adult brain and spatial learning and memory. J. Biol. Chem. 292, 4533-4543 (2017)

19. Chen, X. R. et al. Uhrf2 deletion impairs the formation of hippocampusdependent memory by changing the structure of the dentate gyrus. Brain Struct. Funct. 223, 609-618 (2018).

20. Debacq-Chainiaux, F., Erusalimsky, J. D., Campisi, J. \& Toussaint, O. Protocols to detect senescence-associated beta-galactosidase (SA-betagal) activity, a biomarker of senescent cells in culture and in vivo. Nat. Protoc. 4, 1798-1806 (2009).

21. Montero, J. A. et al. Role of FGFs in the control of programmed cell death during limb development. Development 128, 2075-2084 (2001).

22. Krygier, M., Podolak-Popinigis, J., Limon, J., Sachadyn, P. \& StanisławskaSachadyn, A. A simple modification to improve the accuracy of methylationsensitive restriction enzyme quantitative polymerase chain reaction. Anal. Biochem. 500, 88-90 (2016).

23. Furusawa, T. et al. Down-regulation of nucleosomal binding protein HMGN1 expression during embryogenesis modulates Sox9 expression in chondrocytes. Mol. Cell. Biol. 26, 592-604 (2006).

24. Tone, S. \& Tanaka, S. Analysis of relationship between programmed cell death and cell cycle in limb-bud. Horm. Res. 48(Suppl. 3), 5-10 (1997).

25. Fernández-Terán, M. A., Hinchliffe, J. R. \& Ros, M. A. Birth and death of cells in limb development: a mapping study. Dev. Dyn. 235, 2521-2537 (2006).

26. Salas-Vidal, E., Valencia, C. \& Covarrubias, L. Differential tissue growth and patterns of cell death in mouse limb autopod morphogenesis. Dev. Dyn. 220 295-306 (2001).
27. Mariani, F. V., Ahn, C. P. \& Martin, G. R. Genetic evidence that FGFs have an instructive role in limb proximal-distal patterning. Nature 453, 401-405 (2008).

28. Niswander, L. \& Martin, G. R. FGF-4 and BMP-2 have opposite effects on limb growth. Nature 361, 68-71 (1993).

29. Lorda-Diez, C. I., Montero, J. A., Choe, S., Garcia-Porrero, J. A. \& Hurle, J. M Ligand- and stage-dependent divergent functions of BMP signaling in the differentiation of embryonic skeletogenic progenitors in vitro. J. Bone Miner. Res. 29, 735-748 (2014).

30. Lorda-Diez, C. I., Montero, J. A., Garcia-Porrero, J. A. \& Hurle, J. M. The tumor suppressor BTG1 is expressed in the developing digits and regulates skeletogenic differentiation of limb mesodermal progenitors in high density cultures. Cell Tissue Res. 364, 299-308 (2016)

31. Asou, Y. et al. Coordinated expression of scleraxis and Sox9 genes during embryonic development of tendons and cartilage. J. Orthop. Res. 20, 827-833 (2002).

32. Lorda-Diez, C. I., Montero, J. A., Garcia-Porrero, J. A. \& Hurle, J. M.Divergent differentiation of skeletal progenitors into cartilage and tendon: lessons from the embryonic limb. ACS Chem. Biol. 9, 72-79 (2014).

33. Bostick, $M$. et al. UHRF1 plays a role in maintaining DNA methylation in mammalian cells. Science 317, 1760-1764 (2007).

34. Chimal-Monroy, J. et al. Analysis of the molecular cascade responsible for mesodermal limb chondrogenesis: Sox genes and BMP signaling. Dev. Biol. 257, 292-301 (2003)

35. Furumatsu, T. \& Asahara, H. Histone acetylation influences the activity of Sox9related transcriptional complex. Acta Med. Okayama 64, 351-357 (2010).

36. Liu, C. F., Angelozzi, M., Haseeb, A. \& Lefebvre, V. SOX9 is dispensable for the initiation of epigenetic remodeling and the activation of marker genes at the onset of chondrogenesis. Development 145, dev164459 (2018).

37. Akiyama, H., Chaboissier, M. C., Martin, J. F., Schedl, A. \& de Crombrugghe, B. The transcription factor Sox 9 has essential roles in successive steps of the chondrocyte differentiation pathway and is required for expression of Sox5 and Sox6. Genes Dev. 16, 2813-2828 (2002).

38. Choudhry, $\mathrm{H}$. et al. Targeting microRNAVUHRF1 pathways as a novel strategy for cancer therapy. Oncol. Lett. 15, 3-10 (2018).

39. Jia, Y. et al. Negative regulation of DNMT3A de novo DNA methylation by frequently overexpressed UHRF family proteins as a mechanism for widespread DNA hypomethylation in cancer. Cell Discov. 2, 16007 (2016).

40. Ashraf, W. et al. The epigenetic integrator UHRF1: on the road to become a universal biomarker for cancer. Oncotarget 8, 51946-51962 (2017).

41. Patnaik, D., Estève, P. O. \& Pradhan, S. Targeting the SET and RING-associated (SRA) domain of ubiquitin-like, PHD and ring finger-containing 1 (UHRF1) for anti-cancer drug development. Oncotarget 9, 26243-26258 (2018).

42. Tien, A. L. et al. UHRF1 depletion causes a G2/M arrest, activation of DNA damage response and apoptosis. Biochem. J. 435, 175-185 (2011).

43. Zhou, L. et al. Regulation of UHRF1 by miR-146a/b modulates gastric cancer invasion and metastasis. FASEB J. 27, 4929-4939 (2013).

44. Beck, A. et al. Overexpression of UHRF1 promotes silencing of tumor suppressor genes and predicts outcome in hepatoblastoma. Clin. Epigenetics 10 27 (2018).

45. Ge, T. T., Yang, M., Chen, Z., Lou, G. \& Gu, T. UHRF1 gene silencing inhibits cell proliferation and promotes cell apoptosis in human cervical squamous cell carcinoma CaSki cells. J. Ovarian Res. 9, 42 (2016).

46. Yang, $C$. et al. Inhibiting UHRF1 expression enhances radiosensitivity in human esophageal squamous cell carcinoma. Mol. Biol. Rep. 40 5225-5235 (2013)

47. Ke, F. F. S. et al. Embryogenesis and adult life in the absence of intrinsic apoptosis effectors BAX, BAK, and BOK. Cell 173, 1217-1230.e17 (2018).

48. Jung, $H$. J. et al. The ubiquitin-like with $\mathrm{PHD}$ and ring finger domains 1 (UHRF1)/DNA methyltransferase 1 (DNMT1) axis is a primary regulator of cell senescence. J. Biol. Chem. 292, 3729-3739 (2017).

49. Obata, Y. et al. The epigenetic regulator Uhrf1 facilitates the proliferation and maturation of colonic regulatory T cells. Nat. Immunol. 15, 571-579 (2014).

50. Kim, J. K. Estève, P. O., Jacobsen, S. E. \& Pradhan, S. UHRF1 binds G9a and participates in p21 transcriptional regulation in mammalian cells. Nucleic Acids Res. 37, 493-505 (2009). 